\title{
On the scaling law of JKR contact model for coarse-grained cohesive particles
}

\author{
Xizhong Chen and James A. Elliott* \\ Department of Materials Science and Metallurgy, University of Cambridge, \\ 27 Charles Babbage Road, Cambridge CB3 OFS, UK
}

\begin{abstract}
The computational cost of using discrete element method (DEM) simulations for particulate processes with fine and cohesive particles is enormously large. To overcome this limitation, various coarse-grain DEM models have been developed which use a smaller number of larger sized particles. Although the computational cost is significantly reduced, the accuracy of the simulations depends on the underlying scaling law. We propose a scaling of the JohnsonKendall-Roberts (JKR) contact model for adhesive viscoelastic particles. A scaling law using a single Bond number or Cohesion number criterion is insufficient to keep the motion of the coarse-grained particles the same as the original particles. The scaling law in this work is developed based on mass, momentum and energy conservation, which achieves good consistency between the kinematic characteristics of the coarse-grained and original particles. The simulated effective coefficients of restitution were compared for a range of particle-wall impact velocities and validated against experimental data.
\end{abstract}

Keywords: Discrete element method; coarse-grain model; JKR contact model; cohesive materials; scaling-up

\footnotetext{
${ }^{*}$ Corresponding author. Tel.: +44 1223335987

E-mail address: jae1001@cam.ac.uk
} 


\section{Introduction}

The discrete element method (DEM) has become an important tool to study granular flows in numerous fields of application, including pharmaceutical, chemical, food, ceramic and metallurgical powder processing (Zhu et al., 2008; Guo and Curtis, 2015; Sakai, 2016). In DEM simulations, the motion of each particle is calculated using Newton's second law of motion and therefore detailed contact information relating to particle-particle and particle-geometry is available to facilitate the understanding of the underlying mechanisms in various processes. However, DEM is a very computationally expensive method for modelling realistic industrial processes since of order tens of thousands to millions of particles are required to be simulated to correctly describe such systems, which requires huge computational resources.

To overcome the limitation on the number of simulated particles, researchers have developed a variety of the so-called coarse-grain DEM models (Sakai and Koshizuka, 2008; Lu and Benyahia, 2018). In the coarse-grain DEM, large-sized coarse-grained particles are introduced to represent a group of the original particles in order to reduce the total number of the simulated particles in the system. The concept of a coarse-grained particle is also referred to as an "imaginary large particle", "representative particle" or "parcel" in the literature (Kazari et al., 1995; Radl et al., 2011; Mokhtar et al., 2012; Liu et al., 2013; Chen and Wang, 2014; Lu et al., 2014). Although the computational cost is significantly reduced, the accuracy and reliability of the coarse grain DEM simulations depend crucially on the underlying scaling laws. Utilizing a scaled-up particle size inevitably changes the kinematics of the particles and thus certain material properties must be modified to compensate for this adjustment. Several scaling laws have been suggested for the linear spring contact model, and some other non-cohesive contact models, while there have been many fewer attempts to systematically study cohesive systems (Sakai et al., 2012; Nasato et al., 2015; Thakur et al., 2016). It is anticipated that the scaling law will become more critical when the simulated materials are cohesive since particle size, material stiffness and adhesive properties will considerably affect the particle interaction behaviour (Hærvig et al., 2017).

The Johnson-Kendall-Roberts (JKR) and Derjaguin-Muller-Toporov (DMT) interactions are two commonly used elastic adhesive normal contact models employed in DEM simulations for smooth spherical particles (Johnson et al., 1971; Derjaguin et al., 1975). The JKR theory takes the adhesive force as acting within the contact area and contributing to the deformation of the contacting surfaces, so a larger contact area than that predicted by Hertz theory is obtained. 
Consequently, the JKR theory is appropriate for soft materials with moderate and high surface energy. JKR-based DEM simulations have been widely used to investigate fine powder mixing, agglomeration and breakage of agglomerates (Kempton et al., 2012; Yang et al., 2013; Alizadeh et al., 2018; Chen et al., 2019). Additionally, DMT-based DEM simulations have been applied for studying the entrainment and dispersion of hard and low surface energy materials (van Wachem et al., 2017).

Table 1 lists a summary of the typical coarse-grained models available in the published literature. It can be seen that most of the early studies were developed for non-cohesive gassolid fluidization systems and used a simple linear spring-dashpot model or Hertzian model. It should be noted that correctly modelling the interphase interaction forces is very important for the simulation of gas-solid systems. Hence, most previous research focused on appropriately scaling the interphase interaction forces, while fewer efforts were carried out to consider the scaling of the inter-particles interaction forces. Lu et al. (2014) proposed an equation to scale the restitution coefficient of the coarse-grained system based on the kinetic theory of granular flows. However, the equation should be used with caution, especially when the calculated restitution coefficient is very small. Nasato et al. (2015) found that the dimensionless overlapbased scaling law failed for modelling cohesive materials since no predictive behaviour could be obtained for a shear test simulation with the JKR model. Behjani et al. (2017) developed a Cohesion number-based scaling for the JKR model, however it is not clear how the contact details on the particle scale will change if the proposed scaling law is applied. In this work, we demonstrate how the contact stiffness, viscoelastic adhesive forces and torques will be altered after the particle size is enlarged in the JKR model. A scaling law is then developed on the contact level to achieve a consistency between the kinematics of the coarse-grained particle and those of the original particles. The contact duration, maximum overlap and the effective coefficient of restitution of particle-particle and particle-wall impacts are studied and used to verify the proposed scaling law, which is applicable to both JKR and DMT contact interactions. 
Table 1 Summary of some typical coarse-grained models available in the published literature

\begin{tabular}{|c|c|c|c|}
\hline Authors & $\overline{\text { Contact model }}$ & Scaling method & System studied \\
\hline $\begin{array}{l}\text { Kuwagi et al. (2004); } \\
\text { Kuwagi et al., (2009) }\end{array}$ & $\begin{array}{l}\text { Linear spring- } \\
\text { dashpot and van } \\
\text { der Waals model }\end{array}$ & $\begin{array}{l}\text { Similar particle } \\
\text { assembly }\end{array}$ & $\begin{array}{l}\text { Gas-solid fluidized } \\
\text { bed }\end{array}$ \\
\hline Washino et al. (2007) & $\begin{array}{l}\text { Linear spring- } \\
\text { dashpot }\end{array}$ & Similarity analysis & $\begin{array}{l}\text { Gas-solid fluidized } \\
\text { bed }\end{array}$ \\
\hline $\begin{array}{l}\text { Link et al. (2009) and } \\
\text { Sutkar et al. (2013) }\end{array}$ & $\begin{array}{l}\text { Linear spring- } \\
\text { dashpot }\end{array}$ & $\begin{array}{l}\text { Archimedes and } \\
\text { Reynolds number- } \\
\text { based scaling }\end{array}$ & $\begin{array}{l}\text { Gas-solid spout } \\
\text { fluidized bed }\end{array}$ \\
\hline $\begin{array}{l}\text { Sakai and Koshizuka } \\
\text { (2009) (Sakai and } \\
\text { Koshizuka, 2008; } \\
\text { Sakai et al., 2010; } \\
\text { Sakai et al., 2012; } \\
\text { Sakai et al., 2014) }\end{array}$ & $\begin{array}{l}\text { Linear spring- } \\
\text { dashpot and van } \\
\text { der Waals model }\end{array}$ & $\begin{array}{l}\text { Force balance and } \\
\text { rotational energy } \\
\text { conservation }\end{array}$ & $\begin{array}{l}\text { Gas-solid pneumatic } \\
\text { conveying and } \\
\text { fluidized bed }\end{array}$ \\
\hline $\begin{array}{l}\text { Bierwisch et al. } \\
\text { (2009) }\end{array}$ & $\begin{array}{l}\text { Hertzian and JKR } \\
\text { model }\end{array}$ & $\begin{array}{l}\text { Dimensional } \\
\text { analysis }\end{array}$ & Cavity filling \\
\hline Mokhtar et al. (2012) & $\begin{array}{l}\text { Linear spring- } \\
\text { dashpot }\end{array}$ & $\begin{array}{l}\text { Similar particle } \\
\text { assembly }\end{array}$ & $\begin{array}{l}\text { Gas-solid fluidized } \\
\text { bed }\end{array}$ \\
\hline Lu et al. (2014) & $\begin{array}{l}\text { Linear spring- } \\
\text { dashpot }\end{array}$ & $\begin{array}{l}\text { EMMS-model based } \\
\text { scaling }\end{array}$ & $\begin{array}{l}\text { Gas-solid fluidized } \\
\text { bed }\end{array}$ \\
\hline Nasato et al. (2015) & $\begin{array}{l}\text { Linear spring, } \\
\text { Hertzian and JKR } \\
\text { model }\end{array}$ & $\begin{array}{l}\text { Dimensionless } \\
\text { overlap-based } \\
\text { scaling }\end{array}$ & Simple shear test \\
\hline Thakur et al. (2016) & $\begin{array}{l}\text { Hertz-Mindlin and } \\
\text { Edinburgh elastic- } \\
\text { plastic adhesive } \\
\text { model }\end{array}$ & $\begin{array}{l}\text { Stiffness scales } \\
\text { linearly with particle } \\
\text { size; adhesion force } \\
\text { scales with the } \\
\text { square of particle } \\
\text { size }\end{array}$ & $\begin{array}{l}\text { Powder confined } \\
\text { compression. }\end{array}$ \\
\hline Chu et al. (2016) & Hertzian model & $\begin{array}{l}\text { Velocity and } \\
\text { momentum } \\
\text { conservation }\end{array}$ & $\begin{array}{l}\text { Dense medium } \\
\text { cyclone }\end{array}$ \\
\hline
\end{tabular}




$\begin{array}{llll}\text { Behjani et al. (2017) } & \text { JKR model } & \begin{array}{l}\text { Dimensionless } \\ \text { Cohesion number- } \\ \text { based scaling }\end{array} & \begin{array}{l}\text { Continuous drum } \\ \text { granulator }\end{array} \\ \begin{array}{l}\text { Tausendschön et al. } \\ (2020)\end{array} & \begin{array}{l}\text { Liquid bridge, } \\ \text { capillary force } \\ \text { model and Van der } \\ \text { Waals model }\end{array} & \begin{array}{l}\text { Dimensionless } \\ \text { overlap-based }\end{array} & \begin{array}{l}\text { Gas-solid } \\ \text { scaling; Bond } \\ \text { number-based } \\ \text { neriodic domain }\end{array} \\ & \text { scaling; Stress-based } \\ & \text { scaling } & \\ & & \end{array}$

\section{Coarse-grain JKR model}

\subsection{Preliminary analysis}

In a coarse-grain model, the coarse-grained particle size is set to be several times larger than the original particle size to decrease the number of particles to be tracked in a system. The ratio between the diameter of a coarse-grained particle and an original particle is referred to as the coarse-grain ratio $k$ here,

$$
k=d_{p, C G} / d_{p, o}
$$

where $d_{p, C G}$ is the diameter of the coarse-grained particle and $d_{p, o}$ is the diameter of the original particle. The total mass of the coarse-grained particles in the system can be calculated as follows,

$$
m_{s y s, C G}=N_{p, C G} m_{p, C G}=\frac{\pi}{6} N_{p, C G} \rho_{p, C G} d_{p, C G}^{3}
$$

Since we would like to decrease the number of particles to be tracked, the density of the coarsegrained particle should be the same as that of the original system to ensure mass conservation for the whole system. That is,

$$
m_{s y s, o}=m_{s y s, C G} \text { and } N_{p, o}=k^{3} N_{p, C G} \Rightarrow \rho_{p, o}=\rho_{p, C G}
$$

In order to make the coarse-grained system comparable to the original system, it is necessary to guaranty that the total momentum is the same for both systems, which gives, 


$$
\sum_{i=1}^{N_{p, C G}} m_{p, C G, i} \boldsymbol{u}_{p, C G, i}=\sum_{i=1}^{N_{p, o}} m_{p, o, i} \boldsymbol{u}_{p, o, i}
$$

Following Sakai and Koshizuka (2009), it is assumed in this work that the translational motion of the coarse-grained particle is equivalent to be the average of that of the original particles. The momentum balance equation for the coarse-grained particles can be calculated as,

$$
m_{p, C G} \frac{d \boldsymbol{u}_{p, C G}}{d t}=m_{p, C G} \boldsymbol{g}+\boldsymbol{F}_{\text {contact }, C G}
$$

Combined with the above analysis $\left(m_{p, C G}=k^{3} m_{p, o}\right.$ and $\left.\mathbf{u}_{p, C G}=\mathbf{u}_{p, o}\right)$, it can be seen that the coarse-grained gravitational force, inter-particle contact force are $k^{3}$ times of those of the original particles. That is,

$$
\begin{gathered}
m_{p, C G} \boldsymbol{g}=k^{3} m_{p, o} \boldsymbol{g} \\
\boldsymbol{F}_{\text {contact }, C G}=k^{3} \boldsymbol{F}_{\text {contact }, o}
\end{gathered}
$$

Based on the mass conservation, Eq. 6 is consistent if the density of the coarse-grained particle is kept the same as that of the original particle. The coarse-grained particle contact force needs to be carefully considered because it is also related to the energy conservation of the system. The energy of the coarse-grained system may be dissipated through friction, inelastic collision and work of adhesive peeling.

\subsection{JKR contact model}

According to JKR theory (Johnson et al., 1971), two colliding surfaces jump into contact at point A (shown in Figure 1) where the normal force immediately drops into a certain value, $F_{A}=-8 / 9 F_{C}$, due to van der Waals attractive forces. The velocity of the sphere is then reduced gradually, and the deformation is increased accordingly. The rise of the deformation increases the repulsive force between spheres and the contact force reaches equilibrium at point B. When the total kinetic energy is transferred into the elastic energy of the sphere, the contact deformation reaches a maximum at point $\mathrm{D}$ where the repulsive force is also largest. At this point, the compression process is terminated, and the unloading process begins. The normal displacement then returns to zero overlap at point A where all stored kinetic energy is recovered. However, the spheres remain sticking to each other at this point. As the spheres continue to move apart, the attractive force will reach its maximum value at point $\mathrm{C}$. The force at this point 
$F_{C}$ is called the pull-off force in the JKR model. The contact breaks until the point of separation at S. Johnson et al. (1971) showed the contact force could be calculated as a function of contact area given as,

$$
F_{n}=\frac{4 E^{*} a^{3}}{3 R^{*}}-\sqrt{16 \pi \gamma E^{*} a^{3}}
$$

where $E^{*}$ is the effective Young's modulus given by $\frac{1}{E^{*}}=\frac{1-v_{1}^{2}}{E_{1}}+\frac{1-v_{2}^{2}}{E_{2}}$ where $E_{i}$ and $v_{i}$ $(i=1,2)$ denote Young's moduli and Poisson's ratios of the contacting spheres, respectively, $\gamma$ is the surface energy and $R^{*}$ is the effective radius given by $\frac{1}{R^{*}}=\frac{1}{R_{1}}+\frac{1}{R_{2}}$ where $R_{1}$ and $R_{2}$ are the radii of the two spheres, respectively.

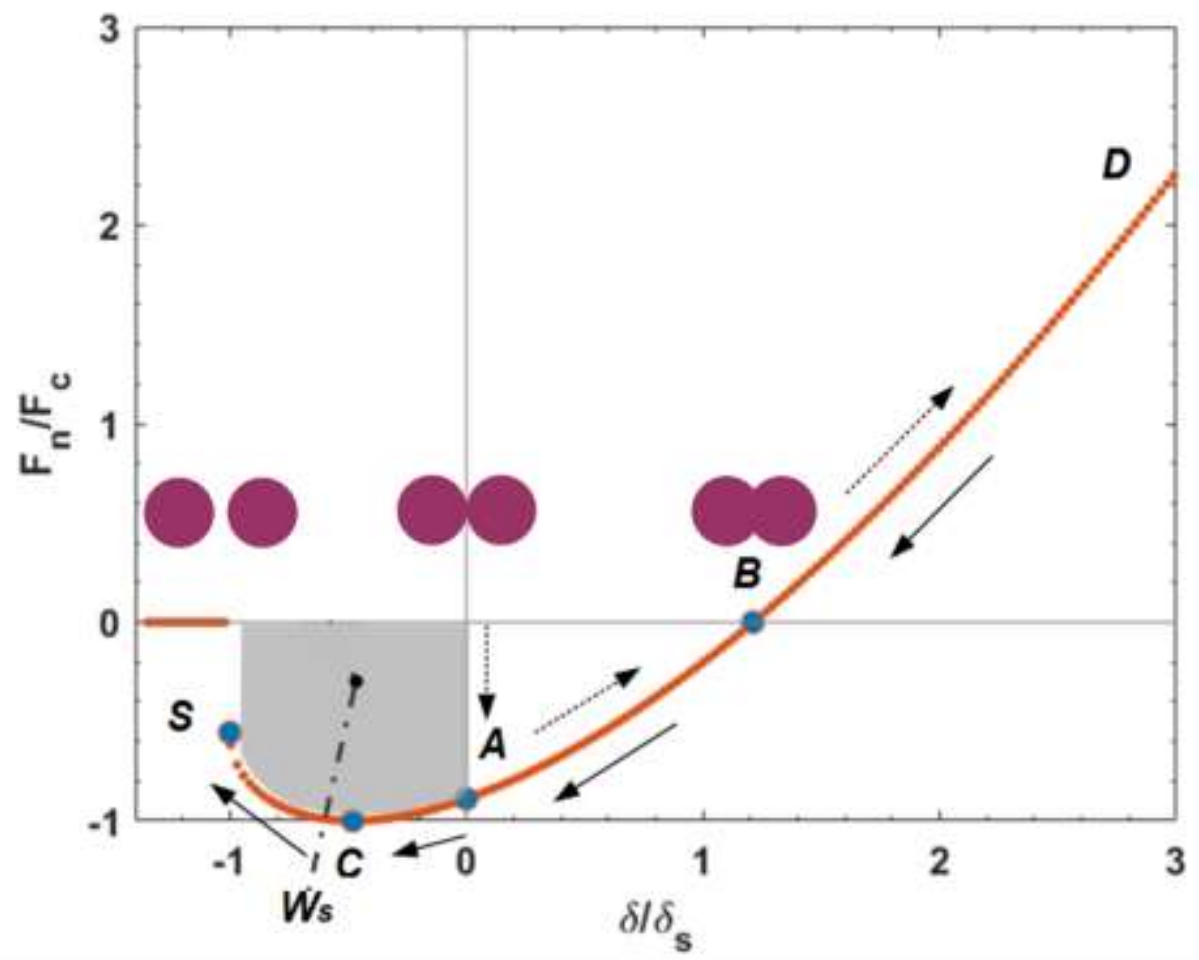

Figure 1. JKR contact force-displacement relationship. Point A: Contact forms and loading begins. Point B: equilibrium position where force is zero. Point D: maximum deformation and unloading begins. Point C: maximum attractive force (pull-off force). Point S: separation point where the contact breaks. Ws: work of adhesive peeling. The relative positions of two colliding spheres are shown in purple.

The contact radius $a$ can be related to the overlap between particles $\delta$, which is given as follows, 


$$
\delta=\frac{a^{2}}{R^{*}}-\sqrt{\frac{4 \pi \gamma a}{E^{*}}}
$$

The forces, overlaps and contact radii at points A, B, C, S can be calculated analytically. They are provided in Table 2.

Table 2. Forces, overlaps and contact radii at points C, S, A, B

\begin{tabular}{|c|c|c|c|}
\hline Point & Force & Overlap & Contact radius \\
\hline $\mathrm{C}$ & $-3 \pi \gamma R^{*}$ & $-0.57\left(\frac{\pi^{2} \gamma^{2} R^{*}}{E^{* 2}}\right)^{1 / 3}$ & $\left(\frac{9 \pi \gamma R^{* 2}}{4 E^{*}}\right)^{1 / 3}$ \\
\hline$S$ & $-1.67 \pi \gamma R^{*}$ & $-1.19\left(\frac{\pi^{2} \gamma^{2} R^{*}}{E^{* 2}}\right)^{1 / 3}$ & $\left(\frac{\pi \gamma R^{* 2}}{4 E^{*}}\right)^{1 / 3}$ \\
\hline A & $-2.67 \pi \gamma R^{*}$ & 0 & $\left(\frac{4 \pi \gamma R^{* 2}}{E^{*}}\right)^{1 / 3}$ \\
\hline B & 0 & $1.44\left(\frac{\pi^{2} \gamma^{2} R^{*}}{E^{* 2}}\right)^{1 / 3}$ & $\left(\frac{9 \pi \gamma R^{* 2}}{E^{*}}\right)^{1 / 3}$ \\
\hline
\end{tabular}

According to Eq. 7 in the preliminary analysis and observing the force formula of pull-off force $\boldsymbol{F}_{C}$ given in Table 2, the scaling ratio for the surface energy can be derived.

$$
\boldsymbol{F}_{C, C G}=k^{3} \boldsymbol{F}_{C, o} \Rightarrow \gamma_{C G}=k^{3} \gamma_{0} \frac{R_{0}}{R_{C G}}=k^{2} \gamma_{0}
$$

It can be seen from Table 2 that once the surface energy is scaled, the forces at points S, A, B are all scaled proportionally. Nevertheless, scaling surface energy ensures that some attractive parts of the contact force are correctly scaled. As shown in Eq. 8, the contact force is also a function of the effective Young's modulus and contact radius. The scaling law for the effective 
Young's modulus is difficult to derive directly from Eq.8 but can be derived from the consideration of energy conservation for the system. Since the JKR loading and unloading paths do not coincide, the work indicated by the shadowed area in Figure 1 is lost. This energy dissipation refers to as the work required to pull two adhered spheres apart to create new surfaces, which is called the work of adhesive peeling (Yin, 1992). This work of adhesive peeling can be quantified by calculating the area above the curve ACS and is given as follows,

$$
\begin{aligned}
W_{s}=\int_{\delta_{A}}^{\delta_{s}} F_{n} d \delta & =\int_{a_{A}}^{a_{s}}\left(\frac{4 E^{*} a^{3}}{3 R^{*}}-4 \sqrt{\pi \gamma E^{*} a^{3}}\right)\left(\frac{2 a}{R^{*}}-\sqrt{\frac{\pi \gamma}{E^{*} a}}\right) d a \\
& =22.5\left(\frac{\gamma^{5} R^{* 4}}{E^{* 2}}\right)^{1 / 3}=0.936 F_{c} \delta_{s}
\end{aligned}
$$

If there are no energy losses due to elastic wave propagation and plastic deformation, the only work dissipated during a collision is the work of adhesive peeling. By applying the energy balances for the coarse-grained particle collision process and that of the original particles, we may write,

$$
\begin{gathered}
\frac{1}{2} m_{o}{ }^{*} u_{r, o}^{2}=\frac{1}{2} m_{o}{ }^{*} u_{i, o}^{2}-W_{S, o} \\
\frac{1}{2} m_{C G}{ }^{*} u_{r, C G}^{2}=\frac{1}{2} m_{C G}{ }^{*} u_{i, C G}^{2}-W_{S, C G}
\end{gathered}
$$

where $u_{i}$ is the impact velocity and $u_{r}$ is the rebound velocity. It can be seen that the work of adhesive peeling needs to be scaled in order to keep the rebound velocity of coarse-grained particle the same as that of original particle, i.e.

$$
W_{S, C G}=k^{3} W_{S, o}
$$

Substituting the formula of the work of adhesive peeling (Eq. 11) into Eq.14, the scaling ratio of the effective Young's modulus can be derived as follows,

$$
E_{C G}^{*}=k^{2.5} E_{o}^{*}
$$

Recalling that the overlap and contact radius are both functions of the material properties (as shown in Table 2), the change of the contact radius and overlap after coarse-graining can be derived. The following relationships are obtained, 


$$
\begin{gathered}
a_{C G}=k^{0.5} a_{o} \\
\delta_{C G}=\delta_{o}
\end{gathered}
$$

A previous attempt at establishing a scaling law for the JKR model assumed that the contact overlap is scaled proportionally to the particle size after coarse-graining (Nasato et al., 2015). However, they found the resulting relationship failed to achieve accurate predictions for shear tests of cohesive materials. The scaling law derived in this work suggests that the overlap should stay the same after coarse-graining while the contact radius is increased by a factor of $k^{0.5}$ during the contact. Substituting the coarse-grained contact radius and other variables into the contact force formula (Eq. 8), we can double-check that the contact force is indeed scaled by a factor of $k^{3}$ in both the attractive and repulsive processes. Since the contact force is scaled by $k^{3}$ while the contact overlap is invariant, we can infer that the contact stiffness is scaled by $k^{3}$. In fact, both the normal and tangential stiffnesses are scaled by $k^{3}$, which can be concluded from the following formula (Mindlin and Deresiewicz, 1953; Thornton and Yin, 1991).

$$
\begin{gathered}
k_{n}=\frac{d F_{n}}{d \delta}=2 E^{*} a \frac{3-3 \sqrt{a_{C} / a}}{3-\sqrt{a_{C} / a}} \\
k_{t}=8 G^{*} a
\end{gathered}
$$

where $G^{*}$ is the effective shear modulus given by $\frac{1}{G^{*}}=\frac{2-v_{1}}{G_{1}}+\frac{2-v_{2}}{G_{2}}$. If the Poisson ratio of the coarse-grained particles is equal to that of the original particles, the effective shear modulus is scaled by $k^{2.5}$. Furthermore, if dashpots are introduced as contact damping to model the energy dissipation due to viscous deformation in the system, the scaling ratio for the coefficient of restitution must be checked. The damping force in normal direction can be calculated as follows,

$$
\begin{gathered}
F_{d}=\eta_{n} \boldsymbol{u}_{n, i j} \\
\eta_{n}=-2 \sqrt{5 / 6} \beta \sqrt{S_{n} m^{*}} \\
S_{n}=2 E^{*} a \\
\beta=\frac{\ln e}{\sqrt{\ln ^{2} e+\pi^{2}}}
\end{gathered}
$$


It can be seen from the following derivation that the coefficient of restitution does not need to be scaled in the coarse-grained system.

$$
F_{d, C G}=k^{3} F_{d, o} \Rightarrow \eta_{n, C G}=k^{3} \eta_{n, o} \Rightarrow \beta_{C G}=\beta_{o} \Rightarrow e_{C G}=e_{o}
$$

As the effective Young's modulus is properly scaled, the spring part of the tangential force is also scaled. Through the Coulomb law $\mu=F_{t} / F_{n}$, it can be seen that the friction coefficient does not need to be scaled in the coarse-grained system because both normal and tangential forces have been scaled at the same time. Similarly, if a rolling friction model is used in the simulation, the rolling friction coefficient does not need to be scaled in the coarse-grained system. So far, we have all the required scaling laws for the material properties in the coarsegrained system. Table 3 lists a summary of the scaling ratios for the material properties in the coarse-grained system.

Table 3. Scaling laws for the coarse-grain JKR contact model

\begin{tabular}{lc}
\hline Property & Scaling law \\
\hline Radius & $R_{C G} / R_{o}=k$ \\
Density & $\rho_{C G} / \rho_{o}=1$ \\
Surface energy & $\gamma_{C G} / \gamma_{o}=k^{2}$ \\
Young's modulus & $E_{C G} / E_{o}=k^{2.5}$ \\
Poisson's ratio & $v_{C G} / \nu_{o}=1$ \\
Friction coefficient & $\mu_{C G} / \mu_{o}=1$ \\
Restitution coefficient & $e_{C G} / e_{o}=1$ \\
\hline
\end{tabular}

\section{Simulation results}

As shown in Figure 2, three cases were designed to test the proposed scaling law, i.e. head-on collision of two particles with different sizes; particle-wall oblique impact and particle-wall normal impact. The first case was used to verify the applicability of the proposed scaling law for a size-disperse system. The second case was used to test if the tangential force and torque were properly scaled as expected. Experimental data of the third case will be used to validate 
the feasibility of the coarse-graining law to predict the effective coefficient of restitution for particle and wall impacts under a variety of collision velocities. The material properties of the simulated cases are listed in Table 4.

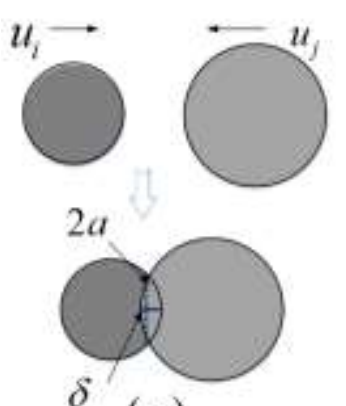

(a)

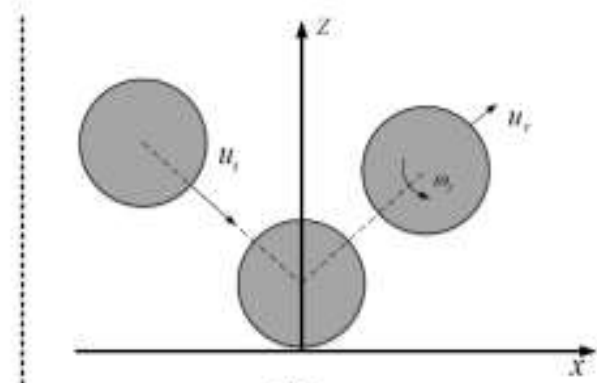

(b)

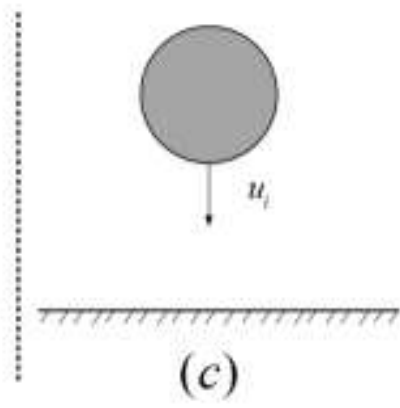

(c)

Figure 2. Diagram of the simulation cases: (a) Head-on impact of unequal-sized particles (b) Oblique impact of a particle with a plane surface (c) Normal impact of a particle with a plane surface.

Table 4. The simulation parameters of the modeling cases

\begin{tabular}{cccc}
\hline Variable & Case a & Case b & Case c \\
\hline Particle size $(\mathrm{m})$ & $5 \times 10^{-4} ; 1 \times 10^{-3}$ & $1.27 \times 10^{-6}$ & $1.27 \times 10^{-6}$ \\
Poisson ratio (-) & 0.3 & 0.3 & 0.3 \\
Young's modulus (MPa) & 1 & $140 ; 98 \times 10^{3}$ & $140 ; 98 \times 10^{3}$ \\
Surface energy $\left(\mathrm{J} \mathrm{m}^{-2}\right)$ & 0.5 & 0.02 & 0.021 \\
Restitution coefficient (-) & 1.0 & 1.0 & 0.97 \\
Friction coefficient (-) & 0.0 & 0.3 & 0.3 \\
Impact velocity $\left(\mathrm{m} \mathrm{s}^{-1}\right)$ & 0.4 & $(-5,0,-7.5)$ & $1 \sim 20$ \\
\hline
\end{tabular}

Figure 3 shows the simulation results of the first test case. It can be seen that the contact mechanics of the coarse-grained particles are in excellent agreement with those of the original particles if the scaling law in this work is applied. The time evolution of post-collision velocities and forces of the coarse-grain system match well with those of the original system. However, the behaviours of coarse-grained particles simulated by using a Bond number scaling criterion and Cohesion number scaling criterion result in significant deviations compared with those of the original particles. Note that the Bond number here is defined as the ratio of 
maximum attractive force and the gravity force (Thakur et al., 2016). The Cohesion number is defined as the ratio of work of adhesive peeling over the particle's gravitational potential energy with regards to a characteristic height equal to particle equivalent radius (Behjani et al., 2017), given as $\operatorname{Coh}=\frac{1}{\rho g}\left(\frac{\Gamma^{5}}{E^{* 2} R^{* 8}}\right)^{1 / 3}$. Figure 3 a shows the velocity evolution of the particle in the left-hand side (the smaller particle shown in Figure 2a), in which all the scaling laws predict a similar trend of the particle velocity. When two particles jump into collision, the contact force is attractive which causes a slight increase of the particle velocity. As the collision continues, the kinetic energy of particle continues to transfer into the elastic strain energy until the particle velocity reaches zero. The unloading process then begins, and particle velocity gradually increases due to the release of the strain energy. The post-collision velocity of the left-hand side particle is larger than the pre-collision because it gains part of momentum from the more massive right-hand side particle. The post-collision velocity is underestimated by the Bond number scaling criterion due to the inappropriate scaling of the contact force as shown in Figure $3 \mathrm{~b}$. It is noted that the contact duration is overestimated by the Bond number scaling criterion which also results in a more considerable maximum contact overlap during the collision, as shown in Figure $3 \mathrm{c}$. This is because the Bond number scaling criterion fails to properly scale Young's modulus, causing a relative smaller contact stiffness, as shown in Figure 3d. Meanwhile, it can also be seen from Figure 3d that the whole JKR contact force-displacement curve is appropriately scaled using the proposed scaling law. In general, The Cohesion number scaling criterion shows improved results than the Bond number scaling criterion but still predicts apparent differences in the microscopic contact details such as the contact duration and maximum deformation. 


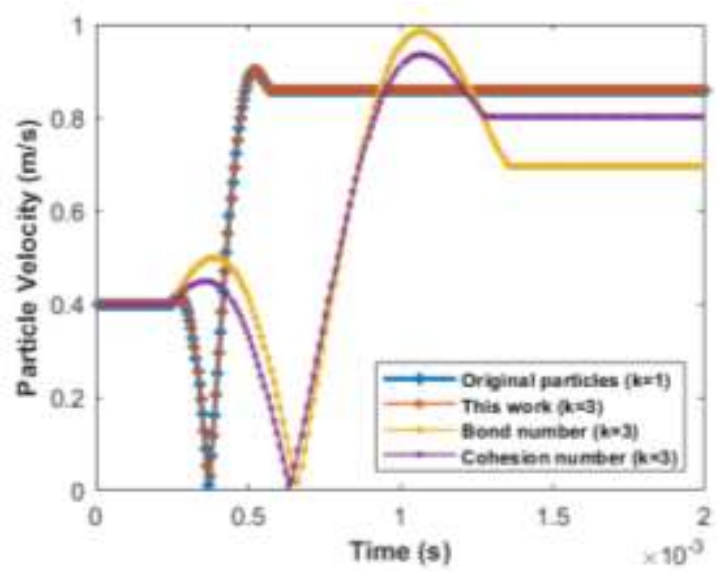

(a)

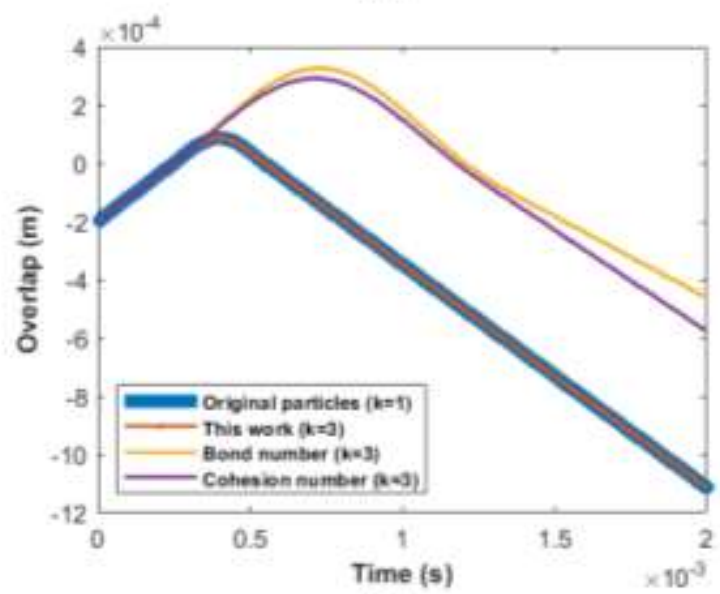

(c)

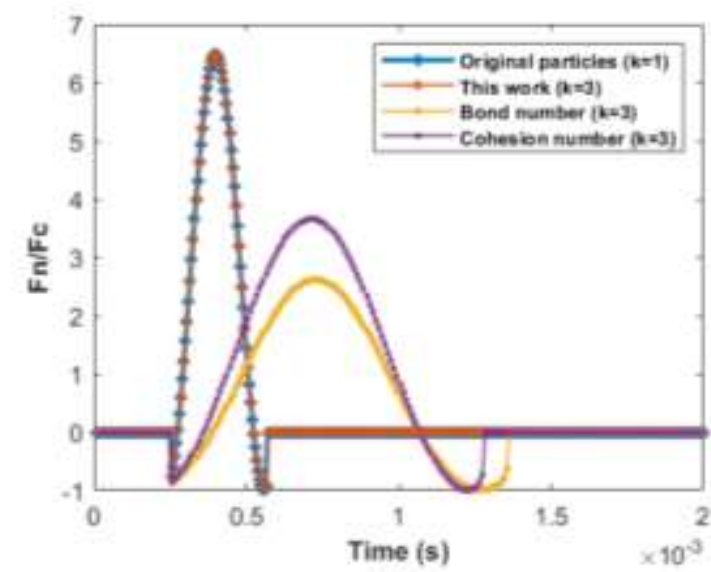

(b)

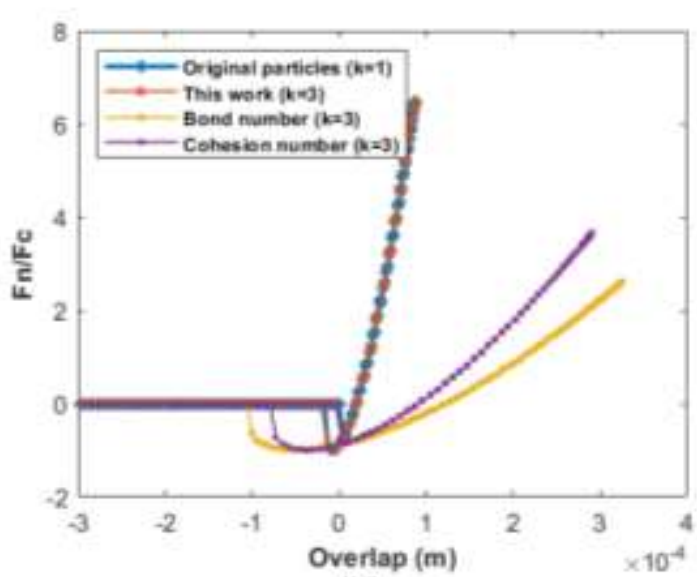

(d)

Figure 3. Comparisons of the contact mechanics for head-on collision of coarse-grained particles simulated using different scaling laws. (a) The time evolution of the velocity of the particle (b) The time evolution of the normal force normalized by the cut-off force (c) The time evolution of the contact overlap (d) The forcedisplacement relations extracted from the simulations.

Figure 4 shows the simulation results of the particle-wall oblique impact case using the scaling law developed in this work. It can be seen that there is good consistency between the coarsegrained system and the original system for contact mechanics. The tangential stiffness, tangential force, rotational velocity and particle velocity are scaled adequately as expected. Note that the current scaling law ensures the translational velocity of the coarse-grained particle is invariant compared with that of the original particle. The rotational velocity at the particle centroid is reduced $k$ times as the coarse-graining ratio increases $k$ times, which is to ensure the tangential overlap between colliding particles is invariant and the rotational kinetic energy is appropriately scaled. This will be further illustrated in the discussion section. 

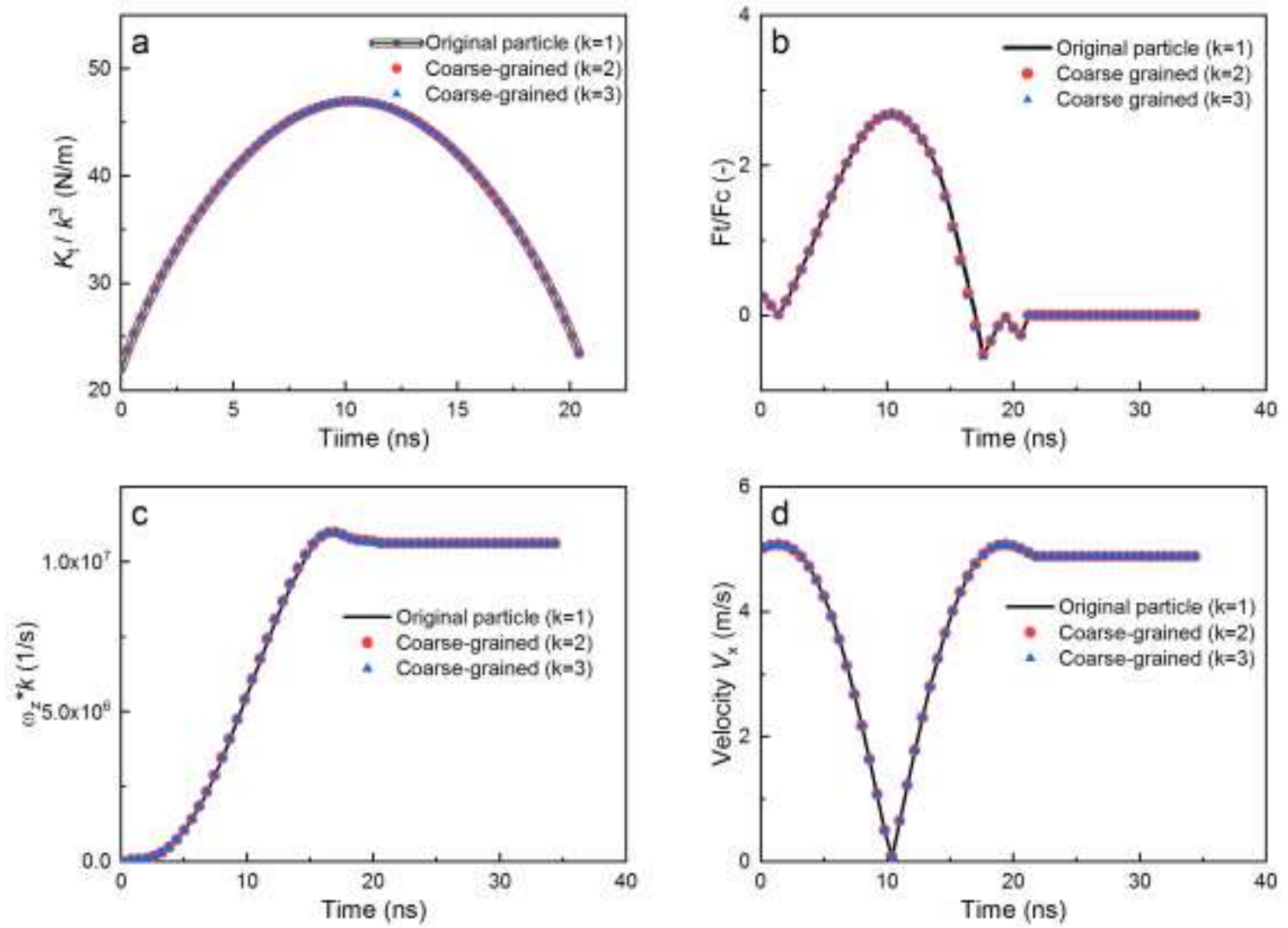

Figure 4. Contact mechanics of particle-wall oblique impact simulated by the scaling law developed in this work. (a) The time evolution of the tangential contact stiffness (b) The time evolution of the tangential force normalized by the cut-off force (c) The time evolution of particle rotational velocity (d) The time evolution of particle translational velocity.

Figure 5 shows the simulation results of particle-wall normal collision against a range of impact velocities in which the coarse-grained simulations using the proposed scaling law all achieve a good match with the experimental data of Dahneke (1975). The decrease of the effective coefficient of restitution at low impact velocity is mainly due to the energy loss caused by the work of adhesive peeling. As shown in Eq. 11, the work of adhesive peeling is fixed for specific material properties and independent of the impact velocity. The significance of this part of energy loss becomes smaller as the particle kinetic energy becomes larger. Therefore, it reaches a plateau when the impact velocity larger than $10 \mathrm{~m} \mathrm{~s}^{-1}$. The plateau of the effective coefficient of restitution at high impact velocities depends on the damping coefficient of the dashpot. The consistency of predictions between different coarse-graining ratios means that the energy due to the work of adhesive peeling and viscous damping are correctly scaled in the coarse-grained simulation. Furthermore, the good agreement between the coarse-grained simulations and experiential results indicates that the material parameters and collision properties of small fine particles could be deduced from experimental measurements on relatively large particles by assuming the scaling properties of the JKR model. Finally, we also checked the maximum 
overlaps, contact durations and energy losses as a function of impact velocity under different coarse-grained ratios. We note that there is an excellent agreement between those coarsegrained simulations and results are not shown here for brevity.

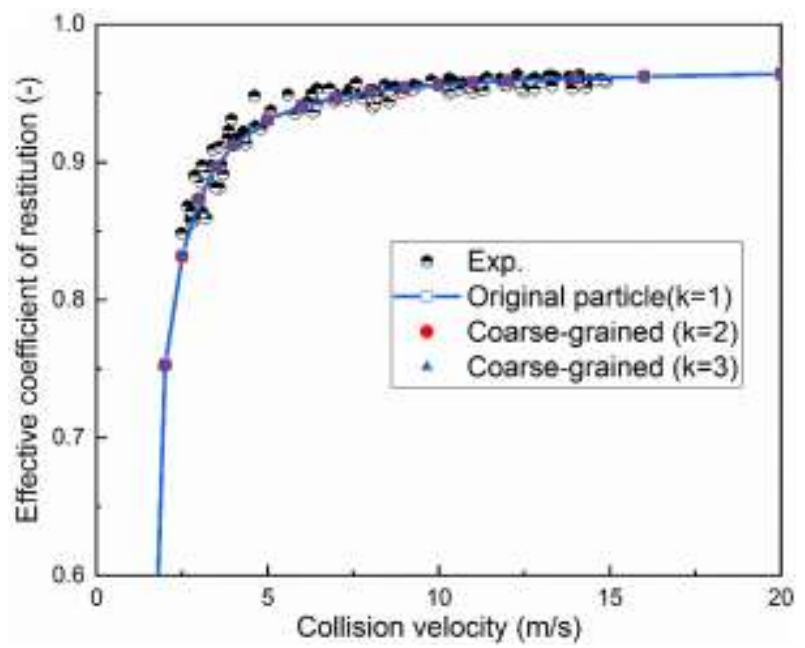

Figure 5. Comparisons between the coarse-grain simulations and experimental results by Dahneke (1975) with spherical particle colliding with a plane wall.

\section{Discussion}

In the above analysis, the translational velocity of the coarse-grained particle is assumed to agree with that of the original particle. This leads to the relative approach between contacting coarse-grained particle maintains the same as that of the original particle. The contact stiffness and contact forces are scaled as $k^{3}$ and the contact torques are scaled as $k^{4}$. As far as the rational motion is concerned, the coarse-grained particle angular velocity is scaled as $k^{-1}$ since the moment of inertia $(I)$ is scaled as $k^{5}$. The scaling of the angular velocity makes the rotational kinetic energy $\left(\frac{1}{2} I \omega^{2}\right)$ between the coarse-grain particle and the group of original particles agree. On the other hand, it also makes the translational velocity of the coarse particle at the contact point $\left(\mathbf{v}_{c}=\mathbf{v}_{i j}+\boldsymbol{\omega}_{i} \times \mathbf{R}_{i c}\right)$ agree with that of the original particle. This ensures that the tangential overlap is correctly scaled during the collision.

$$
\frac{d \boldsymbol{\omega}_{p, C G}}{d t}=\frac{\boldsymbol{R}_{p, C G} \times \boldsymbol{F}_{t, C G}}{I_{C G}}=k^{-1} \frac{d \boldsymbol{\omega}_{p, o}}{d t}
$$

As presented in the simulation results of the first case, a scaling law using the Bond number criterion fails to reproduce the kinematics of the original particles despite the fact that it is usually used in the scaling simulations of cohesive materials (Thakur et al., 2016). The Bond 
number is defined as the ratio of maximum attractive force and the gravity force. For the JKR model, it gives,

$$
B o=\frac{F_{C}}{m g}=\frac{3 \pi \gamma R^{*}}{m g}=2.25 \frac{\gamma}{\rho g R^{* 2}}
$$

It can be verified that the Bond number is invariant after coarse-graining by the scaling law proposed in this work. The Bond number only appropriately scales the ratio of surface energy to particle size. Behjani et al. (2017) argued that the Bond number does not include Young's modulus to account for deformation and they suggested a dimensionless number based on the ratio of work of adhesive peeling over the particle's gravitational potential energy with regards to a characteristic height equal to particle equivalent radius.

$$
\operatorname{Coh}=\frac{1}{\rho g}\left(\frac{\Gamma^{5}}{E^{* 2} R^{* 8}}\right)^{1 / 3}
$$

where $\Gamma$ is the interfacial energy and $\Gamma=2 \gamma$ for an interaction of the same material. However, it can be verified that the Cohesion number defined in this form is not invariant if the scaling law proposed in this work is substituted into Eq.27. Therefore, it cannot ensure that the kinematics of coarse-grained particles agree with those of the original particles on the microscopic scale, which is confirmed in the particle head-on collision simulation results. In fact, if the separation distance of the adhesive peeling (from points $A$ to $S$ ) is used for the characteristic height of gravitational potential energy, the Cohesion number reduced to a similar form of the Bond number and becomes scale-invariant.

$$
\operatorname{Coh}=\frac{W_{s}}{W_{g}}=\frac{0.9355 F_{c} \delta_{s}}{m^{*} g \delta_{s}}=\frac{0.9355 \times 3 \pi \gamma R^{*}}{\frac{4}{3} \pi R^{* 3} \rho g}=2.1 \frac{\gamma}{\rho g R^{* 2}}
$$

Another simple yet effective test of the validity of a scaling law is whether or not it is able to correctly predict the sticking/rebounding problem of fine particles after coarse-graining. The collision-sticking phenomenon among fine particles exists in a variety of areas of engineering, biology and environmental sciences. The question of whether a collision may result in sticking or rebounding is of importance for the formation of particle agglomerates or deposits. When the initial kinetic energy is less than the work of adhesive peeling in the JKR model, the two collision particles will adhere. The critical sticking velocity can be obtained analytically in the 
JKR model and given as $V_{\text {stick }}=\sqrt{\frac{45}{m^{*}}}\left(\frac{\gamma^{5} R^{* 4}}{E^{* 2}}\right)^{1 / 6}$. It can be seen that using only a Bond number or either form of Cohesion number as the scaling law is not enough to predict the critical sticking velocity after coarse-graining. The critical sticking velocity is invariant if the scaling law of this work is applied, which is a significant advantage over the other approaches. From a practical point of view, this ensures the collision dynamics of the coarse-grained particles under low impact velocity regime is the same as that of the original particles, which will be essential to capture agglomeration, de-agglomeration and deposition phenomena.

Besides the JKR model, the DMT theory is another approach that can be used to simulate cohesive materials. The DMT theory assumes that the attractive force does not affect the deformation of the contacting surfaces and considers the adhesive force and Hertz theory separately. Therefore, the DMT theory is more suitable for hard material with low surface energy. In contrast, the JKR theory is appropriate for soft materials with moderate and high surface energy. The appropriateness of choice between JKR and DMT adhesive elastic contact can be characterized by the dimensionless Tabor parameter, defined as (Tabor, 1977),

$$
T a=\left(\frac{4 R \gamma^{2}}{E^{2} \varepsilon^{3}}\right)^{1 / 3}
$$

where $\varepsilon$ is the equilibrium spacing in the Lennard-Jones potential. The parameter Ta was shown by Tabor (1977) to be a measure of the magnitude of the elastic deformation compared with the range of surface forces. The JKR model is suggested to be used for high values of $T a$ and DMT is suitable for low $\mathrm{Ta}$. It can be seen that $\mathrm{Ta}$ is scaling invariant if the scaling law in this work is applied. Moreover, by observing the contact force formula of DMT theory (Derjaguin et al., 1975; van Wachem et al., 2017) and following a similar analysis, it can be demonstrated that the current scaling law is also applicable to the DMT model.

A suitable numerical time step is essential to perform efficient simulations of relatively large systems for relatively long times. The maximum value of DEM simulation time step is dictated by the duration of a contact, i.e. $\delta t=T_{c} / K_{N}$, where $K_{N}$ is the minimum number of steps during one contact duration $T_{c}$ (Van der Hoef et al., 2006). It is recommended that $K_{N}$ must not be less than 5, and is normally set to be a value in the range of 15 50. In the Hertzian contact model, the contact time duration can be derived analytically (Raman, 1920; Seville and Wu, 2016), i.e. $T_{H e r t z, c}=5.09\left(\frac{\rho}{E^{*}}\right)^{2 / 5} \frac{R^{*}}{v_{i}^{1 / 5}}$, where $v_{i}$ is the impact velocity. It can be seen that the contact 
duration is unaffected if the scaling law in this work is used. Note that if the surface energy is zero, the JKR model is reduced to Hertzian model. Unfortunately, there is no analytical solution for the time duration of full JKR model. However, as shown in Figure $3 b$ and Figure $3 c$, the contact duration is scaling invariant in both cohesive and elastic regimes using the proposed scaling law in this work. Additionally, Chen et al. (2015) derived a characteristic collision time for the JKR model, given by $T_{J K R, c}=6.72 \rho^{1 / 2} \gamma^{-1 / 6} R^{7 / 6} E^{-1 / 3}$. It can be verified that this characteristic collision time is invariant if the scaling law of this work is adopted, which is another significant advantage over previous methods. Therefore, the numerical time step of the coarse-grained simulations can be the same as for the original system. As shown in Figure 6, the contact durations are also numerically confirmed to be scaling invariant under a wide range of impact velocities.

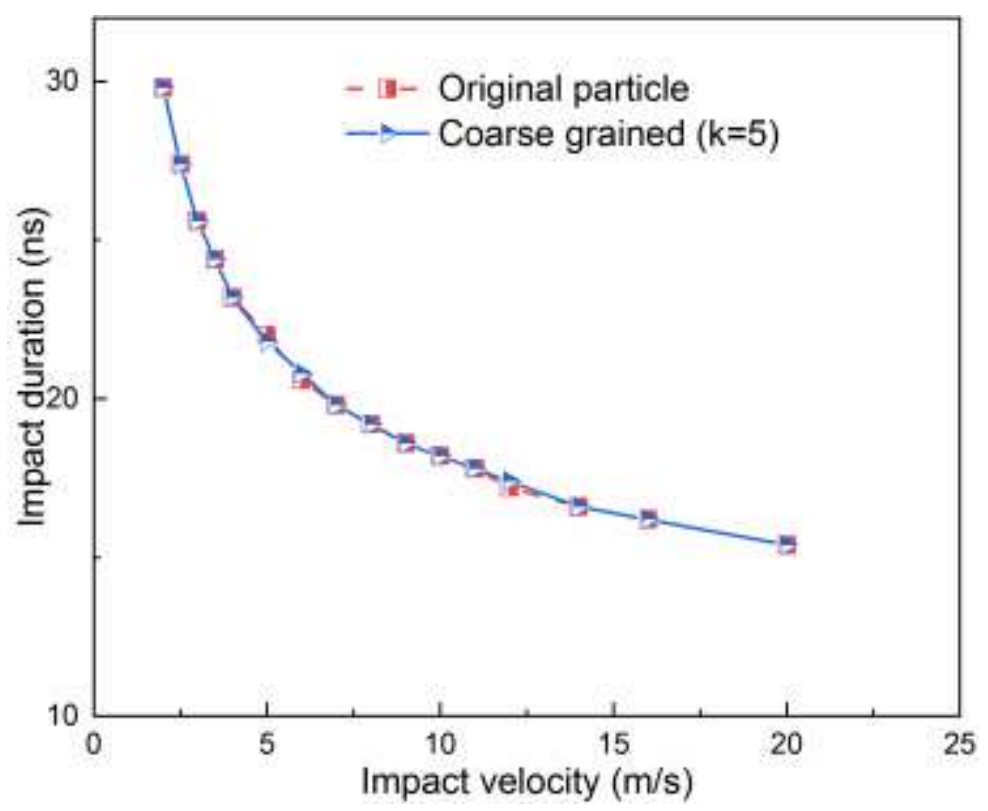

Figure 6 Contact durations under a range of impact velocities for original (red, half-filled squares) and coarsegrained (blue, half-filled triangles) systems. The material parameters are the same as for case 3 in section 2.2.

To verify the feasibility of the proposed scaling law in a many-particle system, simulations of the angle of repose were carried out. First, a hollow cylinder was filled with particles which were allowed to settle under gravity onto a flat planar surface. The cylinder was then lifted with a vertical speed of $0.01 \mathrm{~m} \mathrm{~s}^{-1}$, thus releasing particles from the bottom. A stable pile of particles was formed at the end of each simulation. An elastic-plastic spring-dashpot rolling friction model was adopted (Ai et al., 2011). The number of simulated particles in the original system was 40,000 and a coarse-graining ratio of 2 was used in coarse-grained simulations. Other simulation parameters are given in Table 5. Comparisons between the simulated angle of 
repose using different coarse-grain scaling laws are shown in Figure 7. It can be seen that coarse-grained simulations with scaling by Bond or Cohesion number overestimate the angle of repose since the agglomeration and de-agglomeration rates are not correctly scaled. It can also be seen from case 1 in section 2.2 that the contact duration becomes larger and the repulsive force on an individual particle contact scale is reduced if the Bond or Cohesion number is used, which explains why the coarse-grained particles appear to become more 'sticky', resulting in a higher pile height than for the original system. However, if the scaling law of this work is adopted, the predicted coarse-grained angle of repose matches well with the original system. Furthermore, it is found that the speed-up of the coarse-grained simulation in this case is 7.75 , which significantly reduces the computational cost.

Figure 8 shows the coarse-grained simulation results of a rotating drum with two different operational conditions using the scaling law proposed in this work. The simulated drum is a cylinder with radius $42 \mathrm{~mm}$ and height $20 \mathrm{~mm}$. The particle properties are identical to those listed in Table 5. It can be seen that the dynamical angles of repose predicted by the coarsegrained simulations agree well with simulations of the original systems. The dynamics of the particles can be seen in the supplemental material in which the simulation videos are provided. Finally, it is worth mentioning that the present scaling method implicitly assumes it is homogeneous in a coarse-grain particle, future work may need to consider the microstructure in the coarse-grain model for simulating more complex systems. 


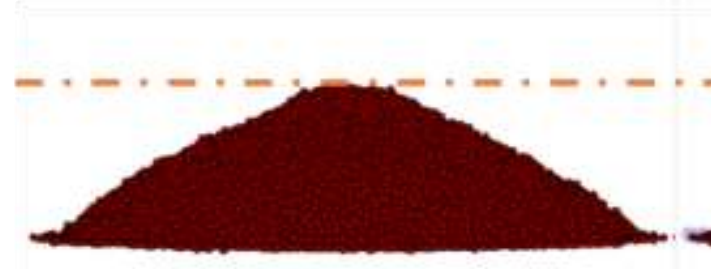

(a) Original system

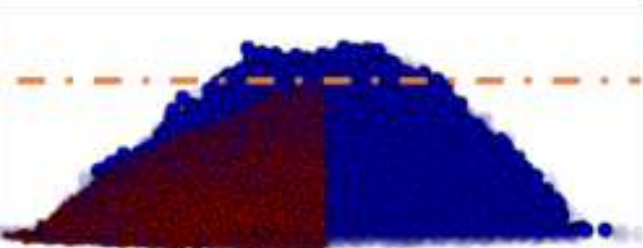

(b) Bond number scaling

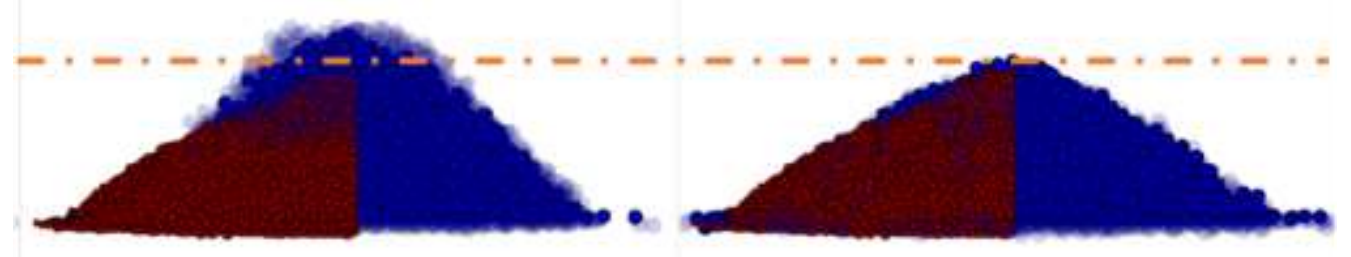

(c) Cohesion number scaling

(d) Scaling by this work

Figure 7. Comparisons between the predicted angle of repose using different coarse-grain scaling laws (b-d) with coarse-graining ratio $k=2$ (blue), and the original system (a) with fine particles (red). The orange dot-dash line indicates the pile height for the original system. For figures (b-d), half of the original system is shown in the left (in red) and overlaid on coarse-grained simulation results (in blue) to aid comparison.

Table 5. Simulation parameters for the angle of repose case

\begin{tabular}{cc}
\hline Parameter & Value \\
\hline Cylinder radius $(\mathrm{m})$ & $1.5 \times 10^{-2}$ \\
Particle radius $(\mathrm{m})$ & $5 \times 10^{-4}$ \\
Density $\left(\mathrm{kg} \mathrm{m}^{-3}\right)$ & 1600 \\
Surface energy $\left(\mathrm{J} \mathrm{m}^{-2}\right)$ & 0.015 \\
Young's modulus (MPa) & 10 \\
Poisson's ratio & 0.3 \\
Particle-particle rolling friction coefficient & 0.3 \\
Particle-wall rolling friction coefficient & 0.3 \\
Particle-particle rolling damping coefficient & 0 \\
Particle-wall rolling damping coefficient & 0 \\
Particle-particle sliding friction coefficient & 0.5 \\
Particle-wall sliding friction coefficient & 0.5 \\
Restitution coefficient & 0.6 \\
Numerical time step (s) & $5 \times 10^{-6}$ \\
\hline
\end{tabular}




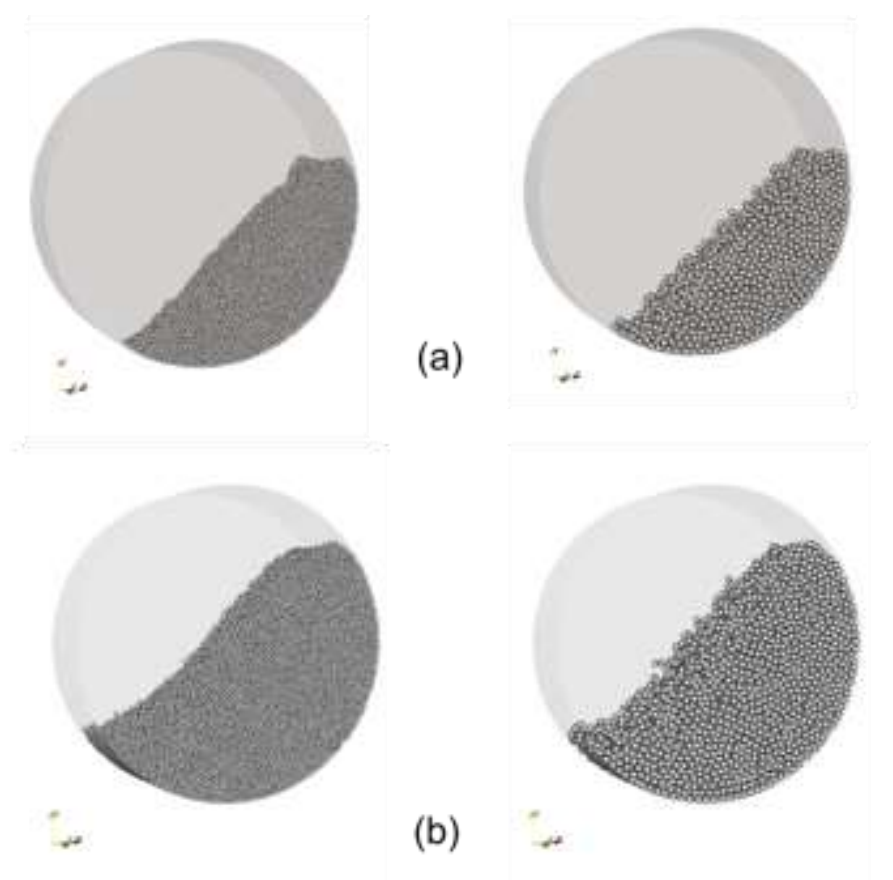

Figure 8. Simulations of a rotating drum with two different operational conditions. (a) $R P M=10$, fill ratio $=$ 0.14 ; (b) $\mathrm{RPM}=20$, fill ratio $=0.28$. Plots on the left are the simulations of the original system (with fine particles) and plots on the right are the coarse-grained simulations with $k=2$.

\section{Conclusions}

In this work, a scaling law for the simulation of coarse-grained cohesive viscoelastic particles interacting via the JKR model was developed. The translational velocity of the coarse-grained particles was assumed to be consistent with that of the group of the original particles, and the respective scale ratios for the material properties were derived. It was found that the Bond number of the coarse-grained system is invariant if the proposed scaling law is applied. However, it was shown that only applying a Bond number criterion or Cohesion number criterion for the coarse-grained simulation is insufficient to ensure that the kinematics of the coarse-grained particles agree with those of original particles. This is because the dimensionless Bond number only considers the scaling of the maximum attractive surface force compared to the gravitational force. Although the Cohesion number includes the effect of elastic modulus, simulation results showed that it still predicts apparently quantitative differences in the microscopic contact details on coarse-grained simulations. The Tabor parameter is another important dimensionless number, representing the ratio of elastic deformation force to the attractive surface force, that is shown to be invariant to coarse-graining under the scaling law in this work. In fact, a combination of Bond number and Tabor parameter 
gives the same scaling ratios of the material properties required in the spring part of the normal force as the scaling law proposed in this work.

Besides scaling the material properties for the normal elastic force, we also derived the coarsegraining ratios for the coarse-grained restitution coefficient and friction coefficient in order to properly scale the dashpot damping force and tangential force. The head-on collision of unequal-sized particles and particle-wall oblique impact cases were simulated to verify the developed scaling law. Simulation results showed that the scaling law proposed in this work could quantitatively reproduce the same velocity evolution as for the original particle. Another appealing feature of proposed scaling law is that the maximum overlap, contact duration and effective coefficient of resolution under a variety of impact velocities were shown to be invariant under coarse-graining. Furthermore, the critical sticking velocity is also invariant under coarse-graining using the current scaling law, which is vital for the coarse-grained simulation of mixing, agglomeration and de-agglomeration of fine powders under a variety of applications. Finally, we demonstrated a simple application by predicting angle of repose for a cylindrical column of particles settling under gravity, and showed that, in contrast to the Bond or Cohesion number criteria, our new scaling law was able to preserve the original shape and height of the particle bed for the coarse-grained particles.

\section{Acknowledgments}

The project is funded through the EPSRC grant INFORM 2020 (EP/N025075/1). The authors would like to acknowledge 3M Ltd., AstraZeneca, GlaxoSmithKline, Malvern Panalytical and the INFORM 2020 Consortium for their support.

\section{References}

Alizadeh, M., Asachi, M., Ghadiri, M., Bayly, A., Hassanpour, A., 2018. A methodology for calibration of DEM input parameters in simulation of segregation of powder mixtures, a special focus on adhesion. Powder Technology 339, 789-800.

Behjani, M.A., Rahmanian, N., Hassanpour, A., 2017. An investigation on process of seeded granulation in a continuous drum granulator using DEM. Advanced Powder Technology 28, 2456-2464.

Chen, S., Liu, W., Li, S., 2019. A fast adhesive discrete element method for random packings of fine particles. Chemical Engineering Science 193, 336-345.

Chen, X., Wang, J., 2014. A comparison of two-fluid model, dense discrete particle model and CFD-DEM method for modeling impinging gas-solid flows. Powder Technology 254, 94-102. Dahneke, B., 1975. Further measurements of the bouncing of small latex spheres. Journal of Colloid and interface science 51, 58-65.

Derjaguin, B.V., Muller, V.M., Toporov, Y.P., 1975. Effect of contact deformations on the adhesion of particles. Journal of Colloid and interface science 53, 314-326. 
Guo, Y., Curtis, J.S., 2015. Discrete element method simulations for complex granular flows. Annual Review of Fluid Mechanics 47, 21-46.

Hærvig, J., Kleinhans, U., Wieland, C., Spliethoff, H., Jensen, A.L., Sørensen, K., Condra, T.J., 2017. On the adhesive JKR contact and rolling models for reduced particle stiffness discrete element simulations. Powder Technology 319, 472-482.

Johnson, K.L., Kendall, K., Roberts, A., 1971. Surface energy and the contact of elastic solids. Proceedings of the royal society of London. A. mathematical and physical sciences 324, 301313.

Kazari, M., Roko, K., Kawaguchi, T., Tanaka, T., Tsuji, Y., 1995. A Study on Conditions for Similarity of Particles Motion in Numerical Simulation of Dense Gas-Solid Two Phase Flow, Proceedings of the 2nd Int. Conf. on Multiphase Flow, Kyoto, Japan.

Kempton, L., Pinson, D., Chew, S., Zulli, P., Yu, A., 2012. Simulation of macroscopic deformation using a sub-particle DEM approach. Powder Technology 223, 19-26.

Liu, Z., Suda, T., Tsuji, T., Tanaka, T., 2013. Use of Similarities in CFD-DEM Simulation of Fluidized Bed. The 14th International Conference on Fluidization - From Fundamentals to Products.

Lu, L., Benyahia, S., 2018. Advances in Coarse Discrete Particle Methods With Industrial Applications, Advances in chemical engineering. Elsevier, pp. 53-151.

Lu, L., Xu, J., Ge, W., Yue, Y., Liu, X., Li, J., 2014. EMMS-based discrete particle method (EMMS-DPM) for simulation of gas-solid flows. Chemical Engineering Science 120, 67-87.

Mindlin, R.D., Deresiewicz, H., 1953. Elastic spheres in contact under varying oblique forces. Journal of applied mechanics 20, 327-344.

Mokhtar, M., Kuwagi, K., Takami, T., Hirano, H., Horio, M., 2012. Validation of the similar particle assembly (SPA) model for the fluidization of Geldart's group A and D particles. AIChE Journal 58, 87-98.

Nasato, D.S., Goniva, C., Pirker, S., Kloss, C., 2015. Coarse graining for large-scale DEM simulations of particle flow-an investigation on contact and cohesion models. Procedia Engineering 102, 1484-1490.

Radl, S., Radeke, C., Khinast, J.G., Sundaresan, S., 2011. Parcel-based approach for the simulation of gas-particle flows, 8th International Conference on CFD in Oil \& Gas, Metallurgical and Process Industries, Trondheim.

Sakai, M., 2016. How should the discrete element method be applied in industrial systems?: a review. KONA Powder and Particle Journal 33, 169-178.

Sakai, M., Koshizuka, S., 2008. Development of a coarse grain simulation methodology for discrete element method in gas-solid flows. Journal of the Society of Powder Technology, Japan 45, 12-22.

Sakai, M., Koshizuka, S., 2009. Large-scale discrete element modeling in pneumatic conveying. Chemical Engineering Science 64, 533-539.

Sakai, M., Takahashi, H., Pain, C.C., Latham, J.-P., Xiang, J., 2012. Study on a large-scale discrete element model for fine particles in a fluidized bed. Advanced Powder Technology 23, 673-681.

Tabor, D., 1977. Surface forces and surface interactions, Plenary and Invited Lectures. Elsevier, pp. 3-14.

Thakur, S.C., Ooi, J.Y., Ahmadian, H., 2016. Scaling of discrete element model parameters for cohesionless and cohesive solid. Powder Technology 293, 130-137.

Thornton, C., Yin, K., 1991. Impact of elastic spheres with and without adhesion. Powder Technology 65, 153-166.

van Wachem, B., Thalberg, K., Remmelgas, J., Niklasson-Björn, I., 2017. Simulation of dry powder inhalers: Combining micro-scale, meso-scale and macro-scale modeling. AIChE Journal 63, 501-516. 
Yang, J., Wu, C.-Y., Adams, M., 2013. DEM analysis of particle adhesion during powder mixing for dry powder inhaler formulation development. Granular Matter 15, 417-426.

Yin, K.K., 1992. Numerical modelling of agglomerate degradation. University of Aston in Birmingham.

Zhu, H., Zhou, Z., Yang, R., Yu, A., 2008. Discrete particle simulation of particulate systems: a review of major applications and findings. Chemical Engineering Science 63, 5728-5770. 